\title{
Ontology Mapping of Design Process Knowledge Based on Classification
}

\author{
Xin Shi, Shurong Tong, Bo Li
}

School of Management, Northwestern Polytechnical University, Xi'an, P.R.China

\begin{abstract}
For the requirements of knowledge reuse in product design process, according to the characteristics of the knowledge representation methods, this paper uses ontology knowledge representation method to construct the product design process knowledge model and gives ontology mapping decision strategy which is based on classification. In the basis of choosing "design department" ontology in human resource management as the heterogeneous ontology of "design organization" ontology in product design process management, lists their concepts set and calculates the similarity of matching concept pairs, finally, outputs the mapping relationship table.
\end{abstract}

Index Terms: Design process knowledge reuse; Ontology; Mapping; Classification; Similarity calculation

(C) 2011 Published by MECS Publisher. Selection and/or peer review under responsibility of the International Conference on E-Business System and Education Technology

\section{Introduction}

Reusing design process knowledge is the precondition of reaching higher-quality and lower-cost in design process. In the knowledge representation, ontology confirms accurate meaning of concepts and their relationships, therefore, ontology is the basis for the information share and reuse.

There are a lot of researches on ontology construction methods at home and abroad. The theory of five ontology construction criterion is more influential which is offered by Gruber. The theory of ontology includes class, hierarchical structure, attributive and relationship which is offered by Natalya. Ontology construction method which is offered by Obrst is similar with Natalya[1]. Previous researches also established ontology models of product design process. However, most ontology models established by using manual pattern and each ontology development team has its own principles and standards, so unification of concepts is mainly realized within a team. Whereas, during the research on the reuse of product design process, the most important thing is the concepts must be coincident and uniform within its field. The optimum method of solving such problem is semantic ontology mapping, which calculates similarity and finds mapping relationship and further establishes the general ontology.

\footnotetext{
* Corresponding author.

E-mail address: hpsx0608@ hotmail.com
} 
For this reason, this paper constructs design process knowledge ontology model, takes "design department" ontology in human resource management as the heterogeneous ontology of "design organization" ontology in product design process management, chooses ontology mapping strategy based on classification and finds their ontology mapping relationship. As a result, it supports to realize more efficient share and reuse of product design process knowledge.

\section{Product Design Process Ontology}

\section{A. The Concept of Product Design Process Ontology}

Definition 1(Product design process ontology): Product design process ontology is a kind of detailed characteristic description of the concepts and relationships which abstracted from the design process, and it is the basis of realizing reuse of knowledge in product design process field [2].

Product design process ontology can be defined as $O_{p r o}\left(C_{p r o}, R_{p r o}\right)$, in which:

- $\quad O_{p m}$ denotes product design process ontology;

- $\quad C_{p o}$ denotes concepts collection of $O_{p w}$;

- $\quad R_{p o v}$ denotes relationships collection of $O_{p o r}$.

\section{B. Product Design Process Ontology Model}

1) Construct the relationship of design process ontology

Through the research of product design process, product design process ontology is divided into design process activity ontology, design process tool ontology, design process result ontology, design process regulation ontology, design process standard ontology, design process experience ontology and design process organization ontology. Part of the concepts and their relationships are shown in Fig. 1.

2) Construct the attributive of design process ontology

In the basis of construction of concept and relationship of product design process, gives the attributive definition of them. To the concept of "organization set", defines the attributives of ID, position name, position constitution and storage. To the concept of "QFD figure", defines the attributives of ID, consent, originate, date, publisher, storage and amendments. To the concept of "plan stage", defines the attributives of ID, target, activity, staff, result, storage, and so on.

3) Fill the example of design process ontology

In the basis of construction of relationship and attributive of product design process, fills the example of product design process and forms the part of product design process ontology as shown in Fig. 2.

\section{Ontology Mapping}

\section{Concept of Ontology Mapping}

Definition 2(Ontology mapping): Ontology mapping refers to confirming how ontology is mapped or mutually related. During exact mapping process, ontology mapping mainly includes 6 kinds of mapping models: 1:1, 1:n, n:1, 1:null, null:1 and n:m [3].

Definition 3(Similarity): The similarity value is among 0 and 1 . $\operatorname{Sim}\left(s_{1}, s_{2}\right)$ is similarity between concept $s_{1}$ and concept $s_{2}[4]$.

- $\quad \operatorname{sim}\left(s_{1}, s_{2}\right)=1$ :denotes concept $s_{1}$ and concept $s_{2}$ are identical; 
- $\quad \operatorname{sim}\left(s_{1}, s_{2}\right)=0$ :denotes concept $s_{1}$ and concept $s_{2}$ are totally different.

\section{Ontology Mapping Based on Classification}

Ontology concept mapping based on natural language disposal is very practical to semantic web, and its basic thought is as follows: first, it searches a group of relevant documents as sample document based on ontology concept; second, it constitutes words eigenvector by considering parameters such as appearance frequency of words in document and their relationships with other words; finally, it calculates similarity of concepts based on concepts eigenvector [5].

In order to reduce complexity of similarity calculation, ontology concept mapping method based on classification is brought out, and its basic thought is as follows: dividing ontology database into several smallsize ontology trees through classification, and establishing similarity mapping on root node; thus, all similarity calculations are established among classification trees where root node of similarity mapping lies. So, the complexity of concepts similarity calculation is reduced significantly [6-8]. The exact procedures are as follows:

\section{1) Give definition}

Basic relationship among concepts is inclusion relationship in ontology database, which is usually described in tree structures. The following definitions are made:

- $\quad O$ and $O$ are two ontology databases mapped;

- $\quad C$ and $C$ are leaf concept nodes of ontology databases;

- $\quad W_{i}$ and $W_{i}$ are classification nodes of ontology databases, and $W_{i} \in O_{1}, W_{i} \in O_{2}$.

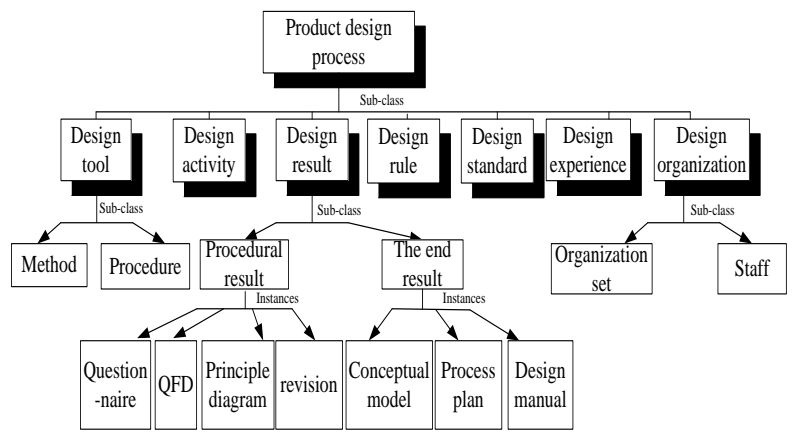

Figure 1. Part of relationship of product design process 


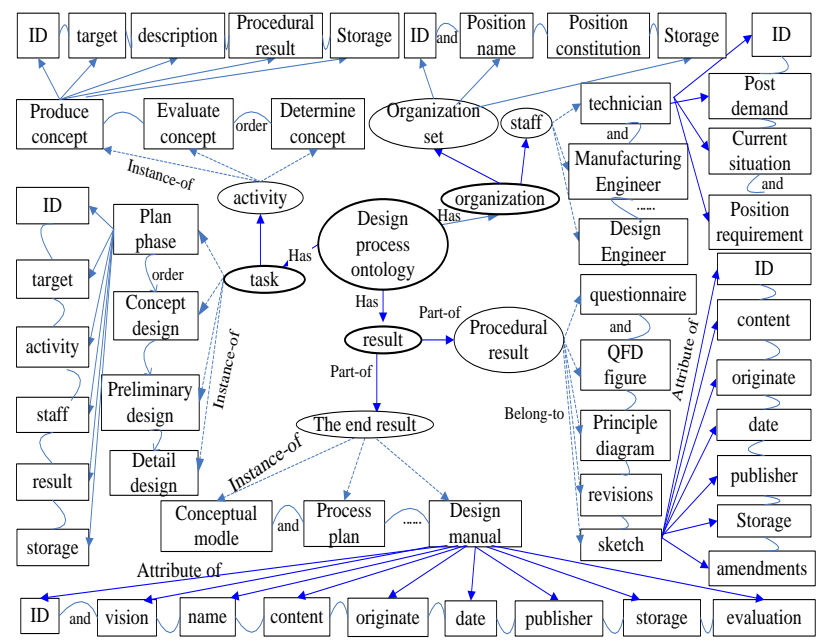

Figure 2. Part of design process knowledge ontology

2) If two classification nodes from the two ontology databases $O_{1}$ and $O_{2}$ are similar, all nodes included in classification tree Tree ${ }_{i}$ and Tree ${ }_{j}$ can be mapped.

3) Procedure of similarity calculation

After mapping relationship of classification nodes is acquired, similarity calculation should be carried out on concept nodes in classification tree which guided by mapping classification nodes [9]. The exact procedures and calculation formula are as follows:

- Take cartesian product of all elements $C_{i} \in$ Tree $_{i}$ and $C_{j} \in$ Tree $_{j}$ as key phrase under classification tree Tree and Tree $e_{i}$, search a sample document for each set of key phrases (e.g. Google search, get the first value), and form a collection $D$, the amount of sample document collection is $K=$ Number $\left(\right.$ Tree $\left._{i}\right) \times$ Number $\left(\right.$ Tree $\left._{j}\right)$.

- Record appearance frequency of possible mapping pair in each sample document as $f_{i j}^{k}$, record appearance frequency of concept $C_{i}$ in sample document as $f_{i}^{k}$,record appearance frequency of concept $C_{i}$ in sample document as $f_{j}^{k}$, then similarity calculation formula can be gotten:

$\operatorname{sim}\left(C_{i}, C_{j}\right)=\sum_{D \in D \text { Mecument }}\left(\frac{2 \times f_{i j}^{k}}{f_{i}^{k}+f_{j}^{k}}\right)$.

Basic thought of (1) is: appearance frequency of mapping pair of which $\operatorname{sim}\left(C_{i}, C_{j}\right) \geq 0.6$ is higher than that of mapping pair of which $\operatorname{sim}\left(C_{i}, C_{j}\right) \leq 0.6$ in sample documents (threshold value of this paper is set to be 0.6, which can be regulated according to actual application field).

4) Concept nodes which can not establish mapping relationship should be calculated according to the traditional similarity calculation method. 


\section{Design Process Ontology Mapping based on Classification}

This paper chooses human resource ontology as the heterogeneous ontology of product design process ontology to establish their semantic ontology mapping relationship.

\section{E. Give Definition}

In ontology database, the basic relationship among concepts is inclusion relationship. Describes relationship between product design process management system and human resource management system with tree structure, and the definitions are as follows:

- $O_{p r o}$ is design process ontology database, $O_{\text {human }}$ is human resource ontology database;

- $C_{i}$ is leaf concept nodes of product design process ontology database, $C_{j}$ is leaf concept nodes of human resource ontology database, and $C_{i} \in O_{p r o}, C_{j} \in O_{\text {miman }}$;

- $W_{i}$ is classification nodes of design process ontology database, and $W_{i}$ is classification nodes of human resource ontology database, and $W_{i} \in O_{p o}, W_{j} \in O_{\text {human }}$;

- $\quad T_{1} e_{1}$ is classification tree of product design process, Tree $e_{2}$ is classification tree of human resource management, and $\exists C_{i} \subset W_{i}, \exists C_{j} \subset W_{j}$.

\section{F. Discover Sharing Knowledge}

Establishes the Correlation matrix of product design process management and human resource management and discovers their sharing knowledge. Fig. 3 shows that "design organization" ontology in product design process management and "design department" ontology in human resource management is possible sharing knowledge while filer out the not related knowledge.

\section{G. Determine Matching Concepts Set}

Takes "design organization" Tree ${ }_{1}$ and "design department" Tree ${ }_{2}$ as the example of classification tree. "Design organization" Tree $e_{1}$ comes from design process ontology database $O_{p r o}$ and "design department" Tree $e_{2}$ comes from human resource ontology database $O_{\text {human }}$.

Concepts set of "design organization" Tree includes: organization set, ID, position name, position constitution, role kind, design engineer, manufacture engineer, technician, product manager, material expert, name, position demand, current situation and position requirement.

Concepts set of "design department" Tree includes: basic attributive, staff ID, name, position, proficiency, position level, position type, design engineer, manufacture engineer, technician, product manager, material expert, state attributive, position constitution, use state and position requirement.

Mapping relationship between "design organization" Tree ${ }_{1}$ and "design department" Tree ${ }_{2}$ will be built by experts in the field, as shown in Fig. 4. 


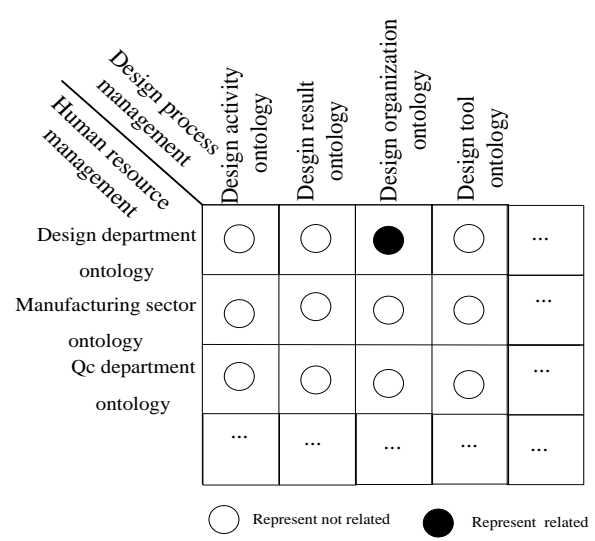

Figure 3. Classification tree of ontology database

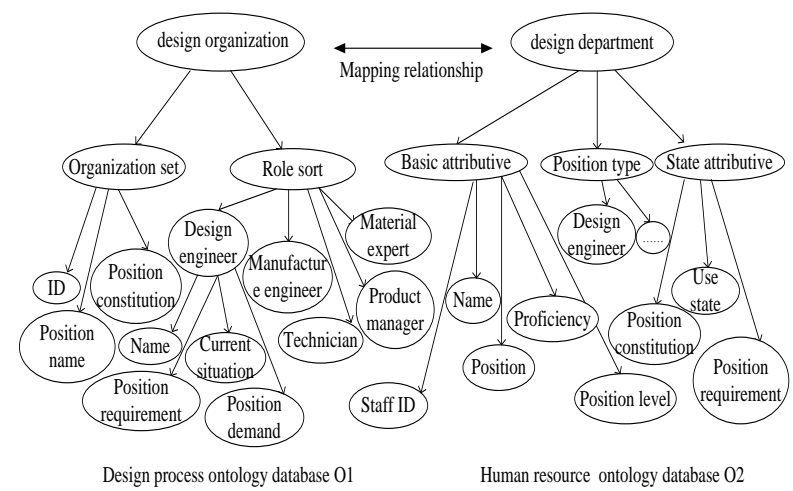

Figure 4. Instances of ontology tree
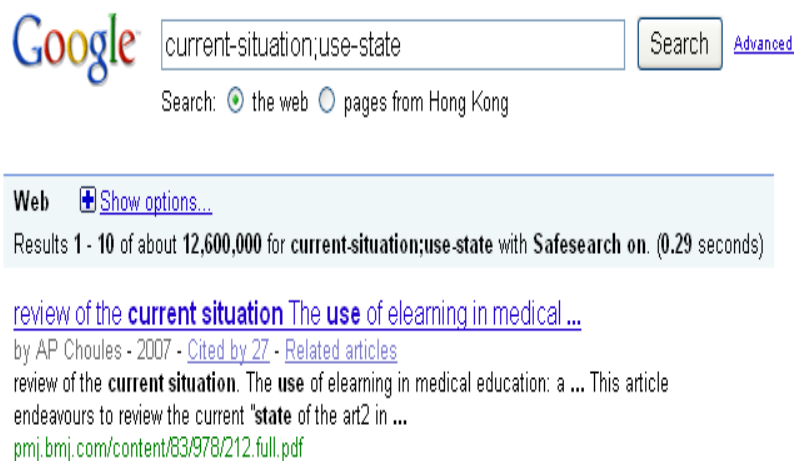

Figure 5. Sample instance of concept mapping documents

\section{H. Mapping Decision}

After acquiring matching concepts set between "design organization" and "design department", the paper carries out similarity calculation of concept nodes in classification tree which guided by mapping classification nodes. 


\section{1) Calculate frequency value of mapping pairs}

Searches a sample document in each possible mapping pairs in "design organization" and "design department" (e.g. Google search, get the first value), and form a sample document collection. Takes (current-situation, usestate) as an example of the mapping pair, exact sample document is shown in Fig. 5.

With appearance frequency of mapping pair(current-situation, use-state)in all sample documents, the paper can acquire appearance frequency $f_{i}^{k}$ of concept "current-situation", appearance frequency $f_{i}^{k}$ of concept "usestate", and appearance frequency $f_{i}^{k}$ of mapping pair (current-situation, use-state) in sample document. Similarly, according to the mapping relationship which experts established, this paper can acquire the appearance frequency $\left(f_{i}^{k}, f_{i}^{k}, f_{i j}^{k}\right)$ of other possible mapping pairs which from concepts in "design organization" and concept in "design department" as shown in Table I.

\section{2) Calculate similarity value of mapping pairs}

According to (1), similarity of mapping pair (current-situation, use-state) can be acquired.

$$
\operatorname{sim}(\text { current }- \text { situation, use }- \text { state })=0.884
$$

Similarly, the similarity value of other concept mapping pairs which from "design organization" ontology and "design department" ontology can be acquired.

Table II shows the similarity of mapping pairs which from concepts in "design organization" ontology and the concept "use-state" in "design department" ontology. Table II also shows that possible concept pairs are (current-situation, use-state) and (post-requirement, use-state) when concept similarity threshold value is 0.6.Actually, the meaning of (post-requirement, use-state) can be estimated, so this kind of pseudo-mapping can be eliminated when experts confirm mapping relationship. Thus, the concept pair with maximum similarity is (current-situation, use-state) between the concepts in "design organization" and the concept "use-state" in "design department".

\section{3) Output mapping relationship}

Similarly, the paper can acquire other similarity value of possible mapping pairs established by experts and find one of which the similarity value is larger than 0.6. The concept pairs with maximum similarity value in two ontology databases are shown in Table III.As the mass calculation,this paper will not list the calculation procedures any more.

\section{Conclusion}

In the basis of product design process knowledge ontology model established, this paper chose ontology mapping method based on classification as decision-making strategy; took "design department" ontology in human resource management as heterogeneous ontology of "design organization" ontology in product design process management; found their ontology mapping relationship; adopted similarity calculation method to carry out similarity of part of mapping pairs. Future work focus on the methods of transforming the knowledge denoted in heterogeneous ontology with mapping relationship for efficient share and reuse of product design process knowledge in a broader scope.

\section{Acknowledgment}

The authors gratefully acknowledge the support of National Nature Science Foundation of China (No. 70771091) and the foundational research project of science and technology industry in national defence (No.Z142008A001). 
TABLE I. FREQUENCY VALUE OF MAPPING PAIRS

\begin{tabular}{|c|c|c|c|}
\hline$\left(\mathrm{C}_{\mathrm{i}}, \mathrm{C}_{\mathrm{j}}\right)$ & $f_{i}{ }^{k}$ & $f_{j}{ }^{k}$ & $f_{i j}{ }^{k}$ \\
\hline (current-situation, use-state) & 23 & 29 & 23 \\
\hline (position-name, use-state) & 6 & 12 & 6 \\
\hline (position-constitution, use-state) & 4 & 9 & 4 \\
\hline (position-requirement, use-state) & 5 & 11 & 5 \\
\hline (ID, use-state) & 19 & 24 & 19 \\
\hline (name, use-state) & 9 & 14 & 9 \\
\hline (position-demand, use-state) & 3 & 7 & 3 \\
\hline
\end{tabular}

TABLE II. SIMILARITY VALUE OF MAPPING PAIRS

\begin{tabular}{|c|c|c|}
\hline Concept in $\mathrm{O}_{1}$ & Concept in $\mathrm{O}_{2}$ & Sim \\
\hline current-situation & use-state & $0.884>0.600$ \\
\hline position-name & use-state & 0.421 \\
\hline position-constitution & use-state & 0.267 \\
\hline position-requirement & use-state & $0.625>0.600$ \\
\hline ID & use-state & 0.131 \\
\hline name & use-state & 0.234 \\
\hline position-demand & use-state & 0.465 \\
\hline
\end{tabular}

TABLE III. CONCEPT PAIRS WITH MAXIMUM SIMILARITY

\begin{tabular}{|c|c|c|}
\hline Concept in $\mathrm{O}_{1}$ & Concept in $\mathrm{O}_{2}$ & Sim \\
\hline current-situation & use-state & 0.884 \\
\hline role-sort & position-type & 0.83 \\
\hline ID & name-ID & 0.61 \\
\hline position-requirement & position-requirement & 1 \\
\hline position-demand & position-level & 0.71 \\
\hline position-constitution & position-constitution & 1 \\
\hline design-engineer & design-engineer & 1 \\
\hline
\end{tabular}

\section{References}

[1] X.Guo and X.G.Li,"Research on Ontology Mapping in Heterogeneous Data Semantic Integration," Software space (in chinese) , 2008,24 (12-3): 272-274.

[2] W.T.Liu, "Research on Ontology-based Heterogeneous Knowledge Integration of Aviation Manufacturing Enterprise,"Academic Paper (in chinese), 2008,17:94 97.

[3] L.P.Zheng,"Design of Ontology Mapping Frame,"Computer Science (in chinese), 2007,34 (10): 165 167.

[4] Z.W.Cao, J. Qian, “An Improved Ontology Mapping Method,'Scientific Technique and Engineering (in chinese),2006,6 (19): $3078 \sim 3082$. 
[5] Q.Fu,"Research on Field Knowledge Share Based on Ontology,"Computer Integrated Manufacturing System (in chinese), 2001,11:54 57.

[6] J.Tang and B.Y.Liang, "Automatic Mapping of Ontology in Semanteme Web,"Chinese Journal of Computer (in chinese), vol.29,no.11,pp.1956 1976,2006.

[7] D.Z.Xu, "Concept Similarity Calculation in Ontology Mapping Process," Computer Engineering and Application (in chinese),2007,43(9):167 169.

[8] X.X.Guo and Z.Yang, "Research on Concept Similarity Calculating Method in Ontology Mapping Based on OWL," Scientific Technique and Engineering (in chinese), 2007, 7 (15): 3761 3765.

[9] K.Q.HE, “Ontology Elements Modeling Theory \& Methods,” (in chinese),Beijing: Science Press, 2008. 